\title{
Adenovirus Expressing the Myostatin-Somatostatin-fusing Gene Increases Mouse Growth Rate
}

\author{
Gaoxiao Xu \\ Nanning University \\ Ning Zhang \\ Jinguang Co.,Ltd \\ Siying Liu \\ Northwest Agriculture and Forestry University \\ Tingting Wei \\ Northwest Agriculture and Forestry University \\ Xingfa Chen \\ DaBeiNong Feed Technology Co.,Ltd \\ Dongling $\mathrm{Yu}$ \\ Nanning University \\ Xinglong Wang ( $\nabla$ wxlong@nwsuaf.edu.cn ) \\ Northwest Agriculture and Forestry University
}

\section{Research article}

Keywords: myostatin, somatostatin, growth rate, muscle mass

Posted Date: December 17th, 2020

DOI: https://doi.org/10.21203/rs.2.19059/v5

License: (c) (i) This work is licensed under a Creative Commons Attribution 4.0 International License. Read Full License 


\section{Abstract}

Background Myostatin (MSTN), a type of transforming growth factor, can negatively regulate skeletal muscle growth. Immunisation of mice with recombinant yeast expressing MSTN increased bodyweight and muscle composition of them. Somatostatin (SST) is an inhibitory effector of growth hormone $(\mathrm{GH})$, and active immunisation against SST with a DNA vaccine improves the growth performance of piglets via an influence on $\mathrm{GH}$ secretion. Here, a recombinant adenovirus was constructed and used to deliver MSTN-SST to mice to achieve the goal of regulation the growth performance of the mice.

Results A recombinant adenovirus, rAd-MSTN-SST, expressing the MSTN-SST fusing protein was successfully rescued in HEK293A cells. The expressions of MSTN-SST were confirmed by western blotting and indirect immunofluorescence. Mice immunised with rAd-MSTN-SST were successfully induced to have an immune response against MSTN-SST, which increased their growth rate and muscle mass. In addition, a booster immunisation was beneficial in terms of higher antibody response and mouse growth rate.

Conclusions Fusing protein MSTN-SST is a good candidate for regulating animal growth rate and muscle mass. Adenovirus can be used as a vector for delivering MSTN and SST to animals.

\section{Background}

Myostatin (MSTN), a member of the transforming growth factor (TGF)- -3 superfamily, is also known as growth and differentiation factor 8 (GDF8) [1]. MSTN has been found to be capable of modulating bodyweight and muscle composition in laboratory and farm animals [2]. Oral feeding mice with recombinant yeast Saccharomyces cerevisiae expressing mammalian MSTN from a plasmid or chromosomal integration gene elicits antigen-specific immune responses, resulting in increased bodyweight and muscle composition [3, 4]. Downregulation of MSTN expression by siRNA or gene knockout also increased muscle mass $[2,5]$. Accordingly, MSTN is an ideal target for regulating animal meat products or human muscle wastage.

Somatostatin (SST) is known to inhibit the release of growth hormone (GH) from the anterior pituitary [6]. Reduction in the concentration of SST in the blood accelerates growth in animals. Immunisation of animals to SST and the resulting induction of related antibodies (anabolic factors) is a means of removing SST's normal inhibitory effects. However, SST is a short peptide hormone with only 14 amino acids and its half-life in the bloodstream is only several minutes. Thus, SST conjugates with various proteins are used for immunisation [7]. Here, MSTN was fused to SST to induce the related immune response, accelerate the growth of animals, and enhance meat products.

Adenovirus vectors are the most commonly employed viral vector for gene therapy and delivery of vaccine antigens. They have been used for the delivery of rabbit haemorrhagic disease virus antigen VP60 [8] and many other virus antigens such as the antigens of porcine reproductive and respiratory syndrome virus [9] and foot-and-mouth disease virus [10]. Immunisation with recombinant adenovirus induces a robust immune response and protects against infection. Here, we used adenovirus to deliver MSTN and SST, which may induce a strong immune response that regulates the growth rate of immunised animals.

\section{Results}

\section{Construction of recombinant viruses}

Shuttle vectors containing the MSTN-SST gene under the control of the CMV early promoter were constructed and verified by sequencing. By recombination with adenovirus backbone vector pAdEasy-1 in BJ5183 cells, recombinant adenoviral plasmids, pAd-MSTN-SST, were obtained. Then, the recombinant plasmids were linearised with endonuclease Pac I and transfected into HEK293A to generate recombinant adenoviruses, rAd-MSTN-SST. After about 10 days' incubation, those recombinant adenoviruses were successfully packaged with a characteristic cytopathic effect in transfected cells. Meanwhile, mocktransfected cells (control samples) retained their singularity. 
The obtained recombinant adenoviruses, rAd-MSTN-SST, were purified three times by the plaque-purified method and titred in HEK293A cells. The titre of the recombinant adenoviruses was $3.6 \times 10^{8} \mathrm{vp} \mathrm{mL}^{-1}$. The expressions of the MSTN-SST proteins were confirmed by indirect immunofluorescent assay (IFA) and western blotting (WB).

Green fluorescence, indicating the expression of MSTN-SST, was observed in rAd-MSTN-SST-infected cells, but not in wtAdinfected cells (Fig. 1A). Correspondingly, the immunoreactive protein bands of MSTN-SST were observed in Lines 1, 2 and 3 (Fig. 1B and sFig 1), loaded with proteins from cells collected at 18, 24, and $36 \mathrm{~h}$ after infection with rAd-MSTN-SST. MSTN-SST expression increased from $2.5 \mu \mathrm{g} / \mu \mathrm{L}$ to $4.1 \mu \mathrm{g} / \mu \mathrm{L}$ and $5.4 \mu \mathrm{g} / \mu \mathrm{L}$ at 24 and $36 \mathrm{~h}$, respectively, after viral infection according to the results of grey scan with Image $\mathrm{J}$,. The related band was not observed in Line 4, in which loaded the proteins from cells infected with wtAd (Fig. 1B and sFig 2).

\section{Vaccination induced humoral immune responses against MSTN-SST}

Mice sera were collected at 7, 14, 21 and 28 days post-immunisation and were detected by ELISA with the $E$.coli-expressed recombinant MST-SST proteins. The recombinant proteins, MSTN-SST, were expressed in E.coli with vector pET32a-MSTN-SST, in which MSTN-SST gene derived from pAd-MSTN-SST was inserted in. The purity and specificity of MSTN-SST were identified by sodium dodecyl sulfate polyacrylamide gel electrophoresis (SDS-PAGE) and reacted with mouse-anti-MSTN polyclonal antibodies and rabbit-anti-SST polyclonal antibodies (1:800 dilutions; Supplementary sFig 3).

The antibody against MSTN-SST was detected as early as 7 days post-inoculation. And reached a peak at day 21 by a single immunisation (Fig. 2). Antibody titres were still increasing at the end of the experiments in group $C$, which was immunised twice. The immunised groups gained significantly high levels of antibody titre since 7 days post-inoculation $(P \leq 0.05)$. Boost immunisation to group $C$ induced significant higher level of humoral response after 14 days (day $28, P \leq 0.05$; Fig. 2) comparing with that from group B.

\section{Bodyweight gain}

All mice were about the same weight with no statistical difference (Fig. 3) at beginning of the experiment. At the end of the experiment, however, they weighed from 34.0 to $40.3 \mathrm{~g}$ and the average weights varied among groups. The bodyweight of each animal was recorded weekly during the 4 experimental weeks. And then the mice were sacrificed.

The average increase in bodyweight of the mice was $14.18 \pm 0.36 \mathrm{~g}$ in Group A, whereas it was $16.70 \pm 0.25 \mathrm{~g}$ in Group B, suggesting that vaccination against MSTN-SST enhanced bodyweight increase. In addition, the increases in bodyweight in Groups $C(19.20 \pm 0.63 \mathrm{~g})$ were significantly higher than those of Group B, suggesting that immunisation boosting was effective, which further confirms the role of immunisation MSTN-SST on growth rate regulation in mice (Fig. 3).

\section{Muscle morphology observation}

To show the effect of the immunisation on mouse muscle, post-mortem examinations were performed. The muscles of immunised mice were more defined and the increased sizes could be recognised by the naked eye (Fig. 4). The weights of the longissimus dorsi (LD) and biceps femoris (BF) were measured and shown in Fig. 5. The average LD and BF sizes in the control group were $0.358 \pm 0.002 \mathrm{~g}$ and $0.239 \pm 0.005 \mathrm{~g}$, respectively. Immunisation increased these to $0.463 \pm 0.004 \mathrm{~g}$ and $0.314 \pm$ $0.002 \mathrm{~g}$ in group $\mathrm{B}$ and $0.494 \pm 0.003 \mathrm{~g}$ and $0.367 \pm 0.005 \mathrm{~g}$ in group $\mathrm{C}$, respectively.

\section{Histological examination}

Histological examination was used to show the micro-level differences of the mice muscle fibres among the groups. The BF muscle fibres of each group were observed under an optical microscope (Fig. 6). Thickened muscle fibres in groups B and $C$ were observed compared with those from group A. In addition, the densities of muscle fibres increased significantly as the numbers of fibres increased significantly. According to the statistical results of ten views with the graphical analysis software Image $\mathrm{J}$, the average proportions of muscle fibres increased from $63.43 \% \pm 3.28 \%$ to $72.18 \% \pm 4.28 \%$ and $83.72 \% \pm 1.67 \%$, respectively. The differences between each group were significant $(P \leq 0.05)$. 


\section{Discussion}

Adenovirus vectors are the most commonly used viral vectors in gene therapy and the delivery of vaccine antigens. Adenovirus vectors used as vaccines are mostly replication-defective, with certain essential viral genes deleted and replaced by a foreign gene expression cassette [11, 12]. Here, the MSTN-SST fusion open reading frame was inserted into the Ad5 genome, which was used to express the protein MSTN-SST. Immunisation with the recombinant adenovirus induced humoral response against the recombinant protein MSTN-SST. The activated immune response against MSTN-SST resulted acceleration of the bodyweight and muscle mass gains in mice. In previous research, MSTN was delivered though a heat-killed whole recombinant yeast $S$. cerevisiae expressing mammalian MSTN from a plasmid, which elicited antigen-specific cell and humoral responses in mice [4]. Immunisation increased bodyweight and muscle composition in mice. Soon after, another report demonstrated that heat-inactivated MSTN-recombinant yeast can promote growth in rabbits and significantly increase muscle development [13]. Then, S. cerevisiae harbouring MSTN in the genome was constructed and used to deliver the MSTN gene to mice by oral immunisation [3], which produced similar results. In addition, monoclonal anti-MSTN antibody injected into the yolk of chicken embryos significantly increased the bodyweight (4.2\%) and muscle mass (5.5\%) of the chicks [14]. Though oral immunisation with $S$. cerevisiae or direct administration of a monoclonal antibody can induce an immune response against MSTN and accelerate growth in animals, such immune operations are difficult to conduct in the field. Immunisation by muscle injection is mostly used in clinical production because it is easy to standardise. Here, adenovirus expression MSTN-SST was given by intramuscular injection and the immunisation induced similar antibody levels.

Animals immunised with SST had increased mean daily weight gain of $10-20 \%$, appetite reduction of $9 \%$, and an $11 \%$ increase in food utilisation efficiency [7]. Improved absorption of food components and slower passage of food through the gastrointestinal tract due to sluggish peristalsis were also observed. Animals immunised with SST and their offspring had correct proportions, and the weight distribution of muscle, bone and fat was the same as in controls [7, 15]. Another study found that immunisation of pregnant goats increased the weight of new-borns by $10 \%$ and increased their milk yield [7]. Here, according to our results, immunisation with MSTN-SST improved the daily weight gain of mice as well as their gross weight. However, we did not measure their food consumption and the mice were allowed to feed freely.

\section{Conclusion}

We constructed an adenovirus which expressed the fusing proteins, MSTN-SST. Mice immunised with this adenovirus had enhanced daily weight gain and muscle mass. This recombinant adenovirus can be used to increase animal meat production and may also be able to treat muscular atrophy disease of human. However, experiments on pigs are needed and serious ethical issues should be considered before such vaccines go on the market.

\section{Methods}

\section{Virus and cells}

The human type 5 adenovirus expression system (replication-defective) was purchased from TaKaRa (Dalian, China). Recombinant adenovirus ( $\mathrm{rAd}$ ) and wild-type adenovirus (wtAd) were grown and titered in HEK-293A cells.

All the cells were cultured in Dulbecco's modified Eagle's medium (DMEM) and supplemented with 10\% foetal bovine serum, 2 $\mathrm{mM} \mathrm{L}$-glutamine, $100 \mathrm{U} / \mathrm{mL}$ penicillin, and $100 \mu \mathrm{g} / \mathrm{mL}$ streptomycin at $37^{\circ} \mathrm{C}$ in a humidified atmosphere of $5 \% \mathrm{CO}_{2}$.

\section{Construction of recombinant adenoviruses (rAd-MSTN-SST)}

The open reading frames of porcine MSTN (GenBank No. AY208121) were amplified by PCR using the primers (MSTN-SSTs and MSTN-SSTa, Table 1) with a plasmid harbouring artificially synthesized MSTN gene (General boil, Co.,Ltd, Anhui ) as templates. SST gene was fused into MSTN by a second round PCR reaction with the primers of MSTN-SSTs and MSTN-SSTa2. The PCR products were cloned into the transfer vector, pAd-shuttle-CMV. The recombinant adenoviral vectors were generated by homologous recombination of linearised transfer vectors with pAdEasy-1 in Escherichia coli BJ5183 strain and confirmed by 
restriction enzyme digestion (New England Biolabs). The recombinant adenovirus was generated by transfection of $1 \mu \mathrm{g}$ plasmid (Pacl linearised) using $3 \mu \mathrm{L}$ of Trans Fast ${ }^{\mathrm{TM}}$ Transfection Reagent (Promega, Madison, USA). When $90 \%$ of the cells showed cytopathic effect, adenoviruses were released by three cycles of rapid freezing and thawing and stored at $-80{ }^{\circ} \mathrm{C}$ after addition of $10 \%$ glycerol.

Table 1 Primers used for amplification MSTN-SST gene

\begin{tabular}{|c|c|c|c|c|}
\hline Name & Sequence $\left(5^{\prime}-3^{\prime}\right)$ & $\begin{array}{l}\text { RE } \\
\text { Site }\end{array}$ & $\begin{array}{l}\text { Products } \\
\text { size }\end{array}$ & $\begin{array}{l}\text { Primers } \\
\text { properties }\end{array}$ \\
\hline MSTN- & & $B g l$ & & Forward \\
\hline SSTs & aggatctatgcaaaaactg & II & & Primer \\
\hline MSTN- & & & $1131 \mathrm{bp}$ & Reverse \\
\hline SSTa & tgagcacccacagcgatctac & & & Primer \\
\hline MSTN- & & EcoR & $1182 \mathrm{bp}$ & Reverse \\
\hline SSTa2 & gatatcctaacaggatgtgaaagtcttccagaagaaattcttgcagccagctgag cacccacagcgatc & V & & Primer \\
\hline
\end{tabular}

$\mathrm{RE}$ : restriction endonuclease

\section{IFA}

IFA was used to identify the expression of MSTN-SST in infected cells. Briefly, HEK293A cells were infected with rAd- MSTNSST or wtAd (wild-type adenovirus) at a multiplicity of infection (MOI) of 5 . After $24 \mathrm{~h}$ incubation, the cells were washed, fixed with $4 \%$ paraformaldehyde $\left(30 \mathrm{~min}\right.$ at $25^{\circ} \mathrm{C}$ ) and incubated with mouse-anti-MSTN (Cloud-Clone Corp., MAB653Po21, 1:800 dilutions) and rabbit-anti-SST polyclonal antibody (Cloud-Clone Corp., PAA592Mu08M, 1:800 dilutions) mixture for $1 \mathrm{~h}$ at $37^{\circ} \mathrm{C}$. Cells were stained with goat anti-mouse and goat anti-rabbit FITC-Conjugated Antibody (Abcam, ab6785, ab6717 1:800 dilutions) for $1 \mathrm{~h}$ at $37^{\circ} \mathrm{C}$. The cells were then washed with PBS and the expression of MSTN-SST was visualised under a fluorescence microscope (Olympus IX73).

\section{WB}

The 293A cells infected with rAd-MSTN-SST were collected at 18, 24 and $36 \mathrm{~h}$ post-infection. The cell lysates were separated by $10 \%$ SDS-PAGE and transferred onto a nitrocellulose membrane (Pall Corporation). The 293A cells infected with wtAd were used as a negative control.

The membrane was incubated overnight in blocking solution (10\% fat-free milk in PBS, PBS-M) at room temperature and incubated with anti-MSTN-SST polyclonal antibodies for $2 \mathrm{~h}$ (Cloud-Clone Corp., MAB653Po21, 1:800 dilutions). The membrane was subsequently reacted for $1 \mathrm{~h}$ with goat anti-mouse $\mathrm{lgG}$ conjugated with horseradish peroxidase (AS003) at a dilution of 1/2000 in PBS-M. Detection was performed using chemiluminescence lumi-nol reagents (Super Signal West PicoTrial Kit, Pierce). By grey scan with Image $\mathrm{J}$ software and comparison with known concentration marker bands, the concentrations of the proteins were calculated according to the obtained grey values of the bands.

$\beta$-actin was used as a reference and the primary antibody against $\beta$-actin was purchased from Boster Co., Ltd. (Wuhan, BM0627).

\section{Immunisation}

Thirty 4-week-old male Chinese Kunming mice were purchased from Chengdu Dashuo Experimental Animal Co. Ltd. and randomly divided into three groups of ten. The mice had very similar weights. The first group received wtAd (at day 0 ) and 
served as a control group (Group A). The second group was given rAd-MSTN-SST at day 0 by intramuscular injection (Group B). The third group was given rAd-MSTN-SST at day 0 and boosted at day 14 (Group C). Mice were kept in cages with an automatic ventilation system. Five mice were kept in each cage and were allowed to feed freely.

The mice were weighed every week and blood was sampled on days 14 and 28. All the mice euthanised by $\mathrm{CO} 2$ inhalation. $\mathrm{CO} 2$ was infused in mice home cage at $10 \%, 30 \%$, or $100 \%$ volume per minute displacement rates. When mice were in deep narcosis, they were sacrificed by cervical dislocation. To observe the muscles, the skins of the mice were removed and the muscle fibres (biceps femoris muscle) were observed by making tissue slices.

\section{Histopathology tests}

The collected biceps femoris muscles were fixed and embedded in paraffin wax and cut into 4-5 $\mu \mathrm{m}$ slices. For microstructural observation, the slices were stained with haematoxylin and eosin. The densities of muscle fibres in ten views were analysed using Image $\mathrm{J}$ software and the average densities of muscle fibre were used to express the increase in muscle quantity.

\section{Enzyme-linked immunosorbent assay (ELISA)}

ELISA plates were coated with the recombinant protein MSTN-SST $(0.2 \mu \mathrm{g}$ per well). The recombinant protein was expressed in E. coli. It was stored at $-80^{\circ} \mathrm{C}$ after purification until assayed. The sera of the mice collected at different times were detected with the ELISA plates as reported [12]. Commercial peroxidase-conjugated rabbit anti-mice IgG (Sigma, Shanghai, China) was used as the secondary antibody. After reaction, the absorbance values of the reaction system at $450 \mathrm{~nm}$ (OD450) were used to represent the antibody levels of the mice. Each sample was assayed in triplicate.

\section{Statistical analysis}

The bodyweights of mice are expressed as means \pm standard deviation (SD) and evaluated with ANOVA. Differences between control and immunised groups were analysed by two-tailed independent Student's $t$-tests. Differences were considered significant at $P<0.05$.

\section{Abbreviations}

MSTN :Myostatin

SST :somatostatin

ELISA: Enzyme-linked immunosorbent assay

IFA: indirect immunofluorescence assay

TGF- $ß$ :transforming growth factor $ß$

GDF8:growth and differentiation factor 8

GH:growth hormone

rAd: recombinant adenoviruses

MOI :multiplicity of infection

PBS: Phosphate Buffer solution

IgG: immunoglobulin G

\section{Declarations}


Ethics approval and consent to participate

All experimental procedures involving animals were reviewed and approved by the Ethics Committee at Northwest A\&F University. Guidelines from the independent Animal Care and Use Committee in Shaanxi Province, China, were strictly adhered to.

\section{Consent for publication}

Not Applicable

\section{Competing interests}

The authors declare no conflict of interest.

\section{Funding}

The construction and identification of the adenovirus was supported by the National Natural Science Foundation of China (No. 31672581) and the Scientific Research and Technology Development Major Project of Nanning $\llbracket 20192004-1 \rrbracket$. The cost of the animials and experimental animal rearing were supported by the Scientific Research and Technological Development Program of Wuming District of Nanning City (20180101). The cost for expression and purification of the antigen MSTN-SST were from a funding of the Basic Ability Promotion Project for Young and Middle-Aged Teachers in Guangxi Universities $₫ 2019 \mathrm{KY} 0941 \rrbracket$. The cost of the equipment and other reagent were paid by the Guangxi Innovation-Driven Development Project\Gui Ke AA181180217囚and the Research project of Nanning University (2018XJ36).

\section{Authors' contributions}

GX and NZ conducted the experiment. SL and TW made the figures and revised the manuscript. XC and DY did the data analysis and XW prepared the manuscript. All authors have read and approved the manuscript.

\section{Acknowledgements}

We would like to show our thanks to Xiang Biao who gave us much help for making the histology examination.

\section{References}

1. McPherron AC, Lawler AM, Lee S-J: Regulation of skeletal muscle mass in mice by a new TGF-beta superfamily member. Nature 1997, 387(6628):83-90.

2. Boman IA, Klemetsdal G, Nafstad O, Blichfeldt T, Våge DI: Impact of two myostatin (MSTN) mutations on weight gain and lamb carcass classification in Norwegian White Sheep (Ovis aries). Genet Sel Evol 2010, 42(1):4.

3. Zhang T, Yang H, Wang R, Xu K, Xin Y, Ren G, Zhou G, Zhang C, Wang L, Zhang Z: Oral administration of myostatin-specific whole recombinant yeast Saccharomyces cerevisiae vaccine increases body weight and muscle composition in mice. Vaccine 2011, 29(46):8412-8416.

4. Zhang T, Sun L, Xin Y, Ma L, Zhang Y, Wang X, Xu K, Ren C, Zhang C, Chen Z et al: A vaccine grade of yeast Saccharomyces cerevisiae expressing mammalian myostatin. BMC Biotechnol 2012, 12(1):97.

5. Bhattacharya TK, Shukla R, Chatterjee RN, Dushyanth K: Knock down of the myostatin gene by RNA interference increased body weight in chicken. J Biotechnol 2017, 241:61-68.

6. Patel YC: Somatostatin and Its Receptor Family. Front Neuroendocrinol 1999, 20(3):157-198.

7. Lunin VG, Sergienko OV, Bader LB, Karpov VA, Tikhonenko TI: Chimeric somatostatin containing protein and encoding DNA, plasmids of expression, method for preparing chimeric protein, strain-producers, immunogenic composition, method for increasing the productivity of farm animals. In. United States; 1994. 
8. Wang X, Qiu L, Hao H, Zhang W, Fu X, Zhang H, He S, Zhang S, Du E, Yang Z: Adenovirus-based oral vaccine for rabbit hemorrhagic disease. Vet Immunol Immunopathol 2012, 145(1):277-282.

9. Cao J, Wang X, Du Y, Li Y, Wang X, Jiang P: CD40 ligand expressed in adenovirus can improve the immunogenicity of the GP3 and GP5 of porcine reproductive and respiratory syndrome virus in swine. Vaccine 2010, 28(47):7514-7522.

10. Sreenivasa BP, Mohapatra JK, Pauszek SJ, Koster M, Dhanya VC, Tamil Selvan RP, Hosamani M, Saravanan P, Basagoudanavar SH, de los Santos T et al: Recombinant human adenovirus-5 expressing capsid proteins of Indian vaccine strains of foot-and-mouth disease virus elicits effective antibody response in cattle. Vet Microbio/ 2017, 203:196-201.

11. Wold WSM, Toth K: Adenovirus Vectors for Gene Therapy, Vaccination and Cancer Gene Therapy. Current gene therapy 2013, 13(6):421-433.

12. Wang X, Tang Q, Qiu L, Yang Z: Penton-dodecahedron of fowl adenovirus serotype 4 as a vaccine candidate for the control of related diseases. Vaccine 2019, 37(6):839-847.

13. Liu Z, Zhou G, Ren C, Xu K, Yan Q, Li X, Zhang T, Zhang Z: Oral administration of myostatin-specific recombinant Saccharomyces cerevisiae vaccine in rabbit. Vaccine 2016, 34(20):2378-2382.

14. Kim YS, Bobbili NK, Paek KS, Jin HJ: Production of a Monoclonal Anti-Myostatin Antibody and the Effects of In Ovo Administration of the Antibody on Posthatch Broiler Growth and Muscle Mass. Poultry Sci 2006, 85(6):1062-1071.

15. Reichlin S, . Secretion of somatostatin and its physiologic function. J Lab Clin Med 1987, 109(3):320-326.

\section{Figures}



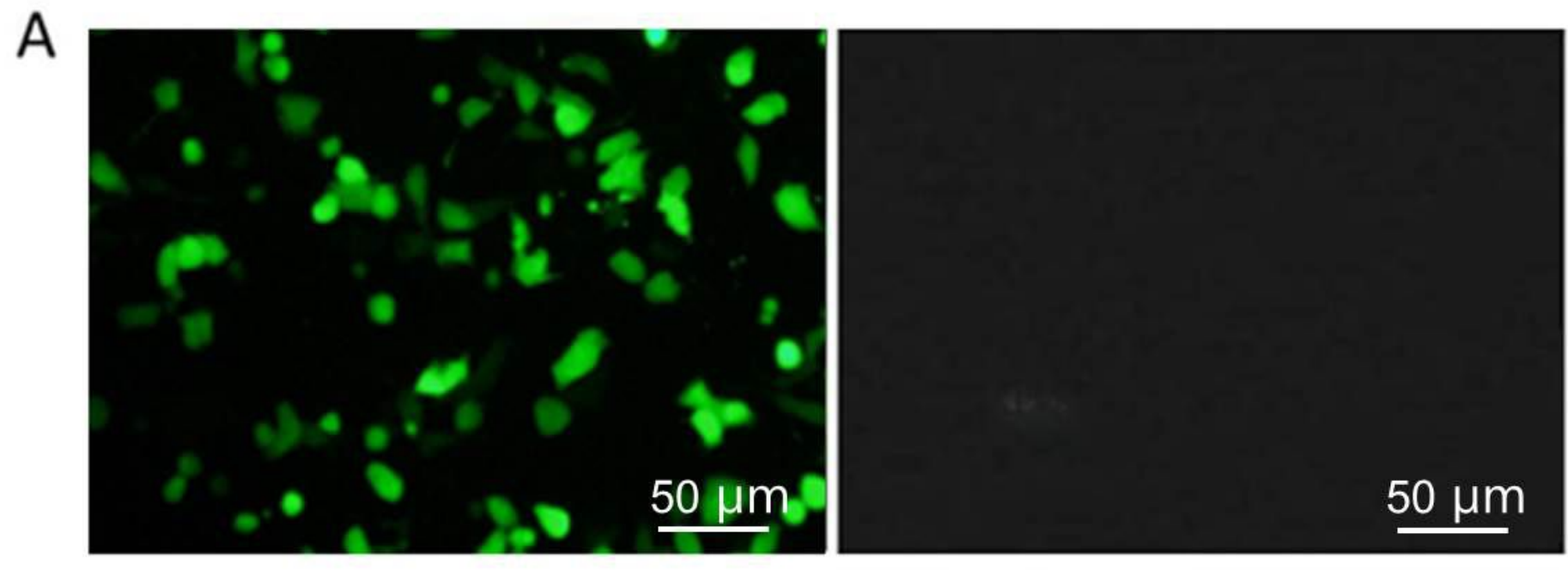

B

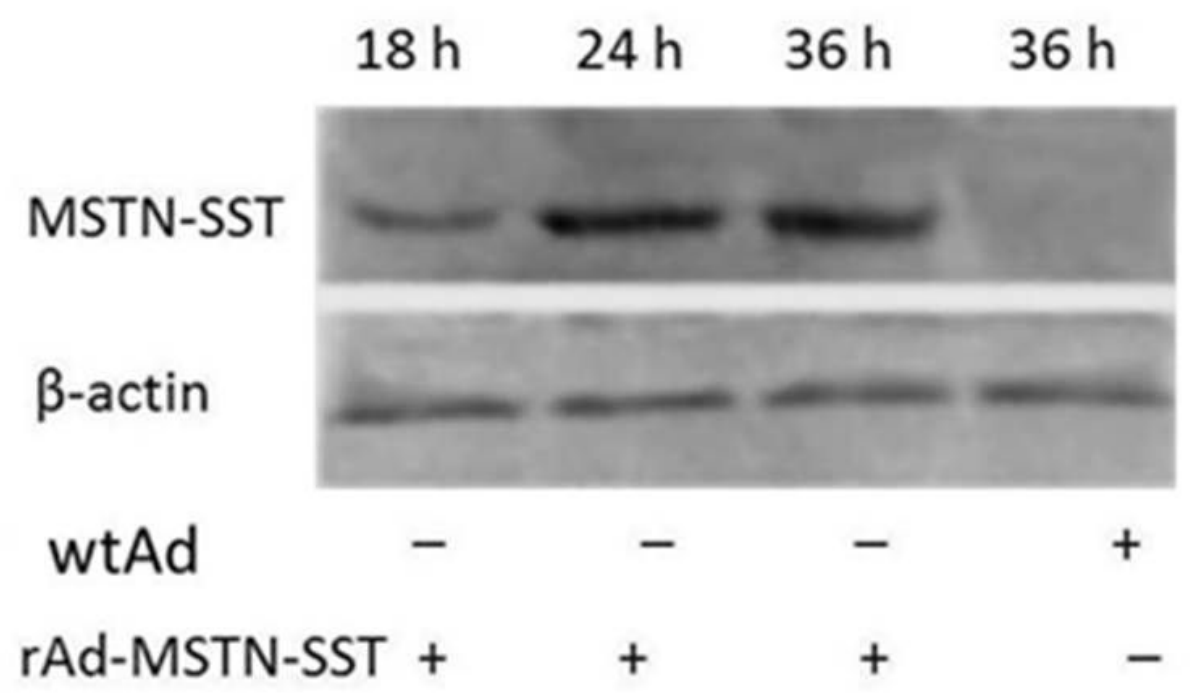

Figure 1

Detection the expression of MSTN-SST using IFA (A) and WB (B). (A) The cells were fixed, and then stained with mouse antibody against MSTN-SST. Positive signals were showed with FITC-conjugated goat-anti-mouse antibody. (B) Target bands of MSTN-SST were detected in cells infected with rAd-MSTN-SST (Lane 1, 2 and 3), but not that of the cells infected with wtAd (Lane 4). 


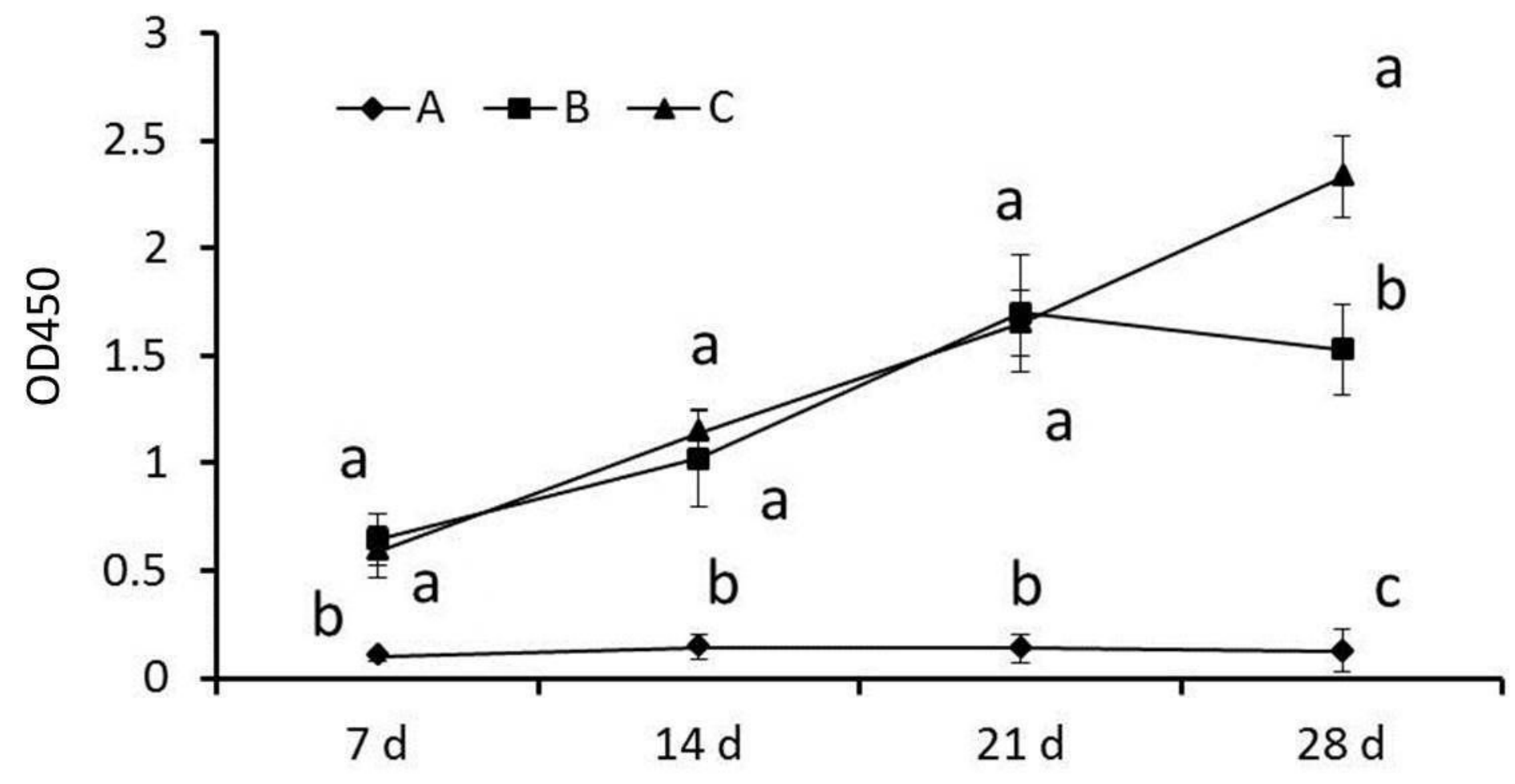

Figure 2

Detection antibody responses against MSTN-SST. The sera were collected at 7, 14, 21 and 28 days post inoculation $(n=10)$. The date collected from group $A, B$, and $C$ were shown as $A, B$ and $C$ in the figure. Data are shown as mean \pm SD. Significant differences were marked with different letter $(a b c)$ and when $p \leq 0.05$ the difference is significant. 


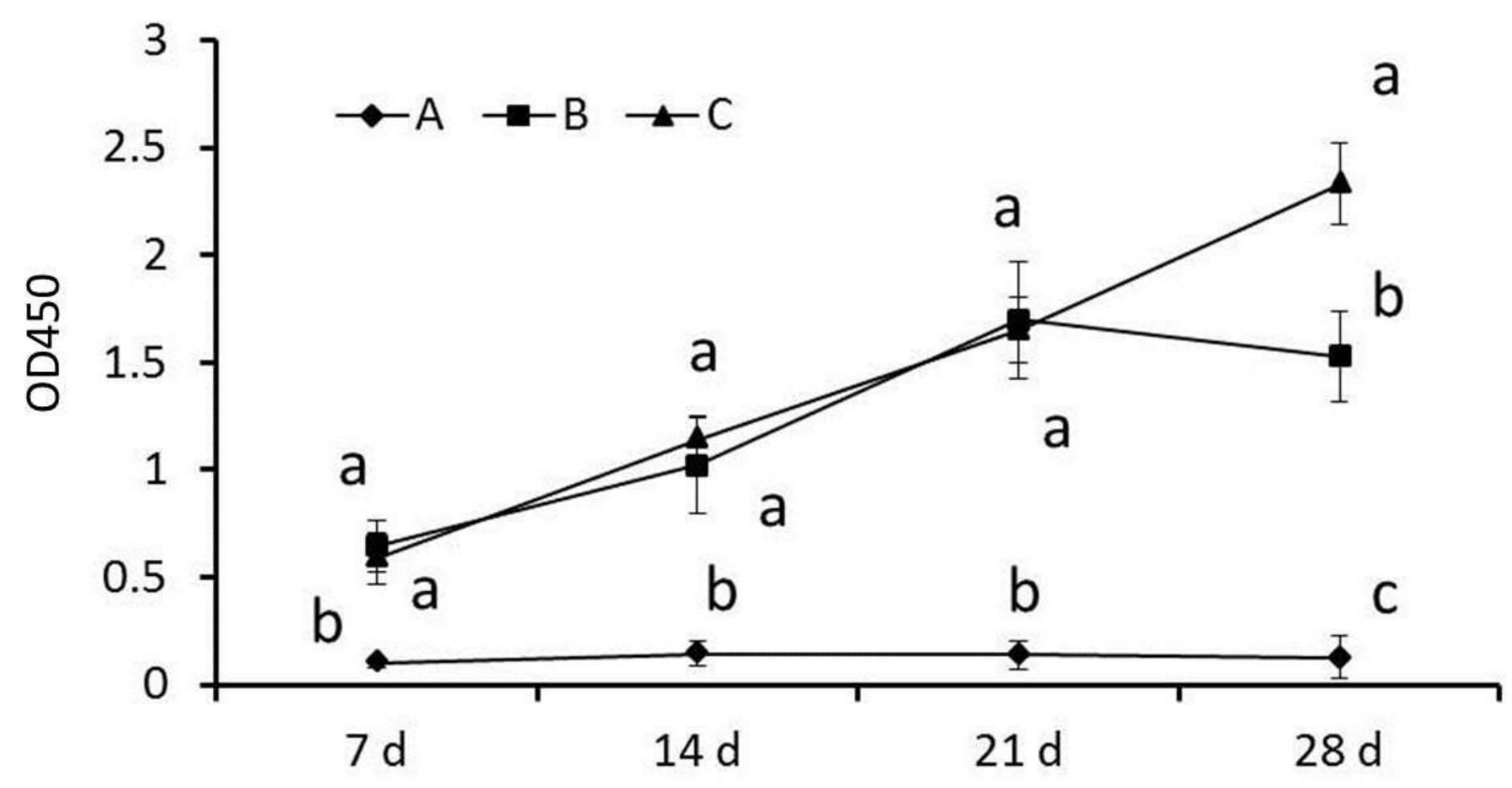

Figure 2

Detection antibody responses against MSTN-SST. The sera were collected at 7, 14, 21 and 28 days post inoculation $(n=10)$. The date collected from group A, B, and C were shown as A, B and C in the figure. Data are shown as mean \pm SD. Significant differences were marked with different letter $(a b c)$ and when $p \leq 0.05$ the difference is significant.

$\mathrm{g} \quad \square \mathrm{A} \quad \square \mathrm{B} \quad$ 目C

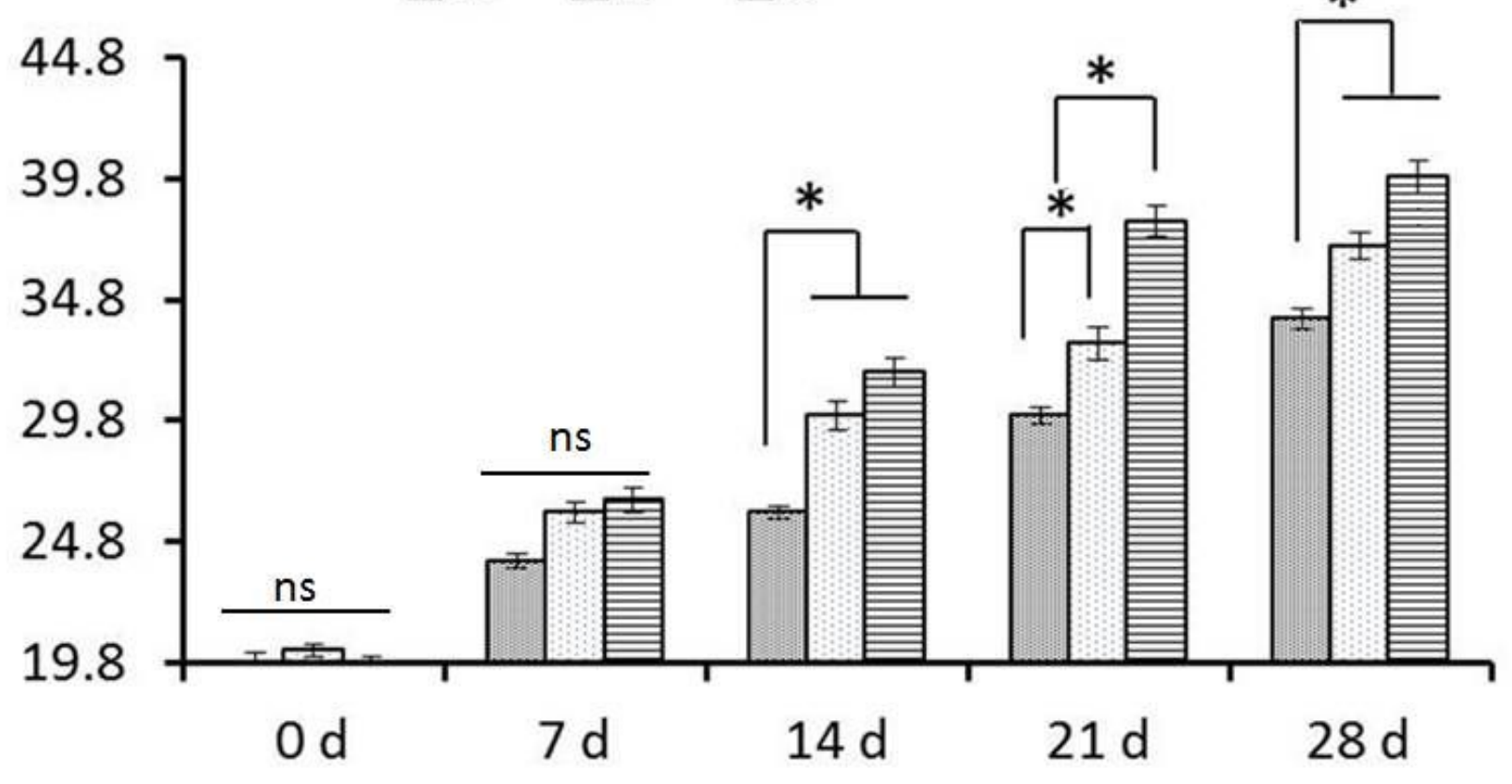


Figure 3

Body weight developments of mice during the experiment. The mice were weighed every week after immunization (weighted three times for each mice). The mean body weight of the mice in each group $(n=10)$ was compared. The date collected from group $A, B$, and $C$ were shown as $A, B$ and $C$ in figure. ${ }^{*}$ was used to show the significant difference $(p \leq 0.05)$. ns: means no significant difference. Data are shown as Mean \pm SD

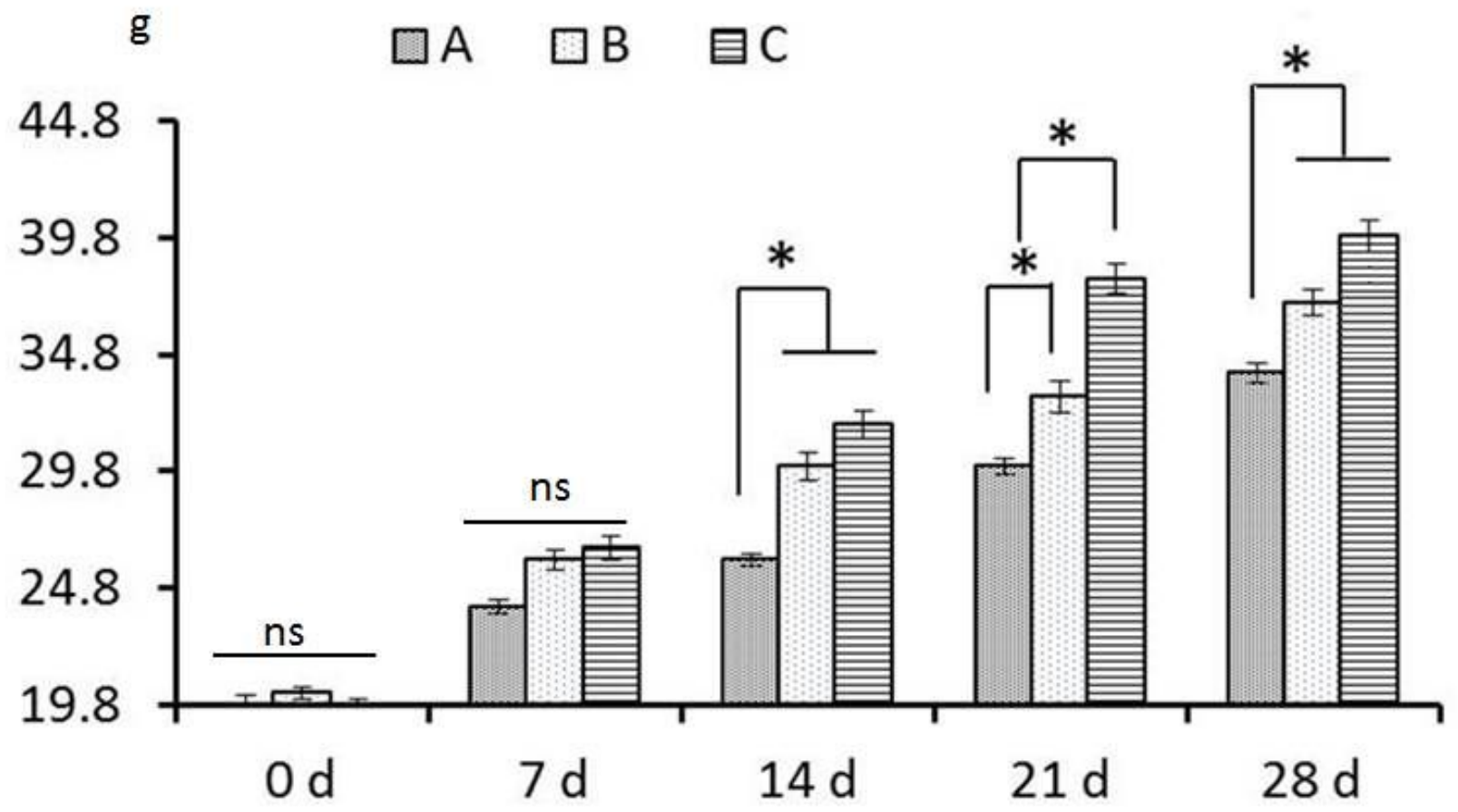

Figure 3

Body weight developments of mice during the experiment. The mice were weighed every week after immunization (weighted three times for each mice). The mean body weight of the mice in each group $(n=10)$ was compared. The date collected from group $A, B$, and $C$ were shown as $A, B$ and $C$ in figure.* was used to show the significant difference $(p \leq 0.05)$. ns: means no significant difference. Data are shown as Mean \pm SD 


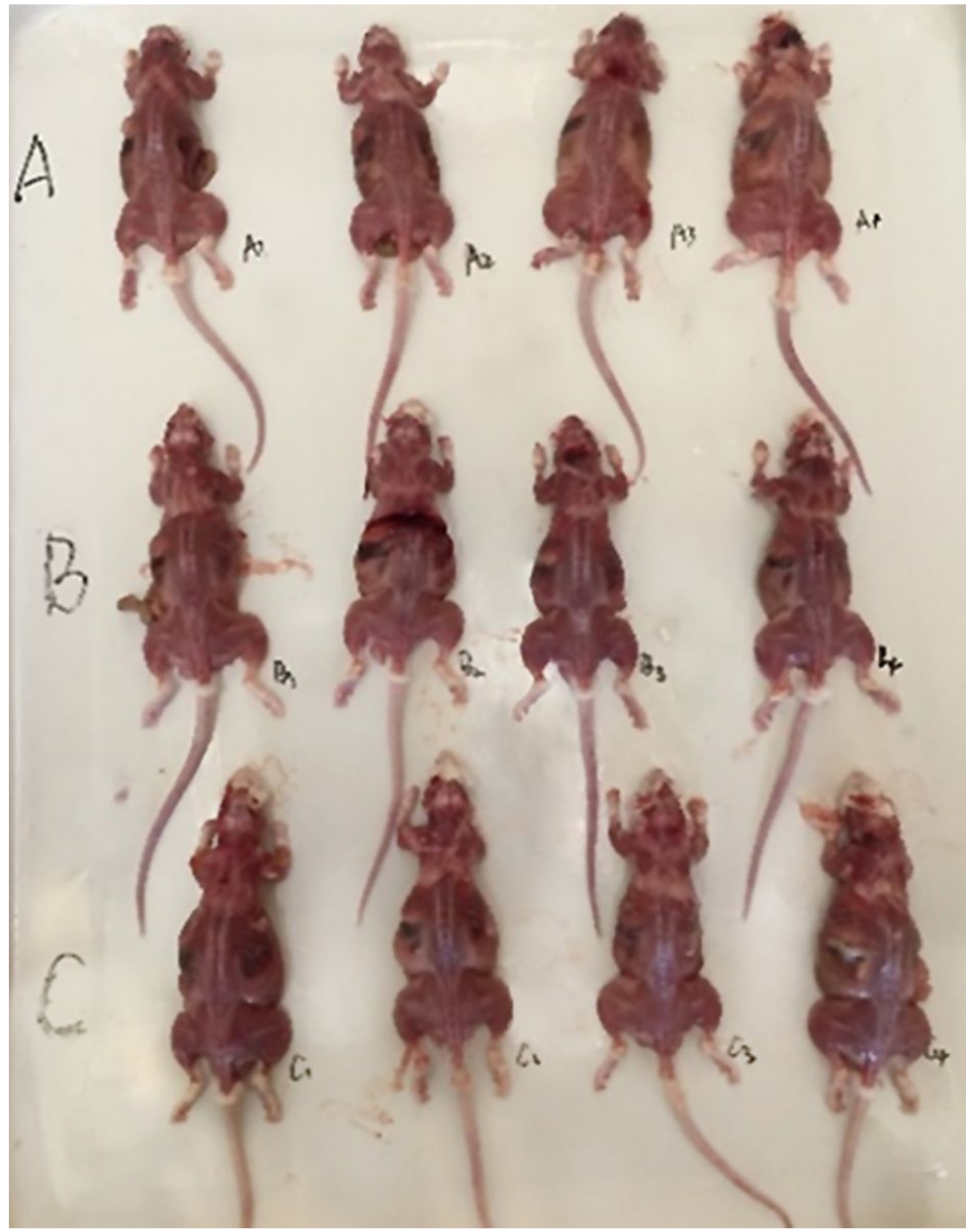

\section{Figure 4}

Observation skeletal muscle morphology of the experimental mice. Random selected four mice from each group were used to show the muscle shape $(n=4)$. The skins of the mice were removed after given euthanasia. The date collected from group $A, B$, and $C$ were shown as $A, B$ and $C$ in the figure. The differences of the muscle between the groups can be visually seen. 


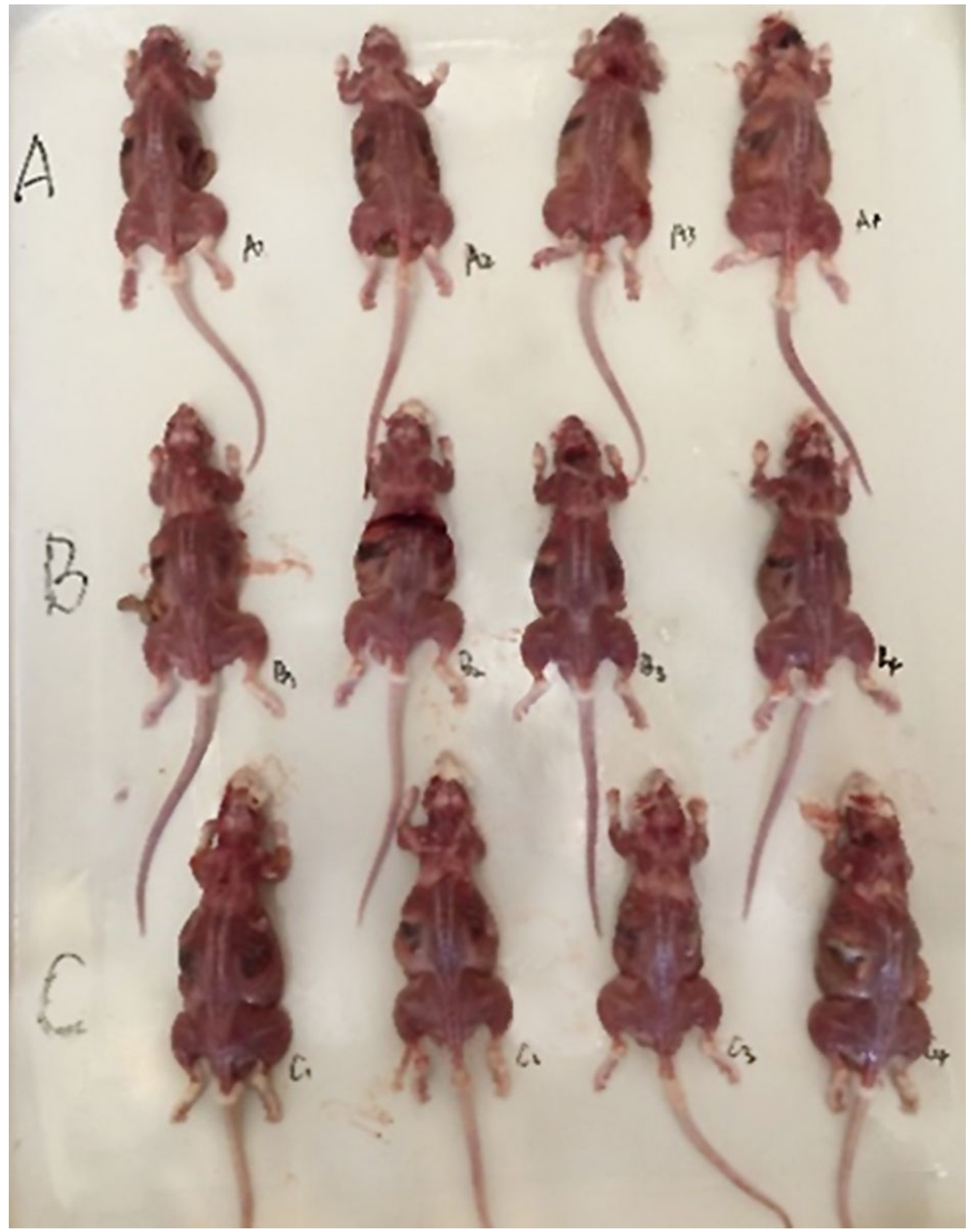

\section{Figure 4}

Observation skeletal muscle morphology of the experimental mice. Random selected four mice from each group were used to show the muscle shape $(n=4)$. The skins of the mice were removed after given euthanasia. The date collected from group $A, B$, and $C$ were shown as $A, B$ and $C$ in the figure. The differences of the muscle between the groups can be visually seen. 


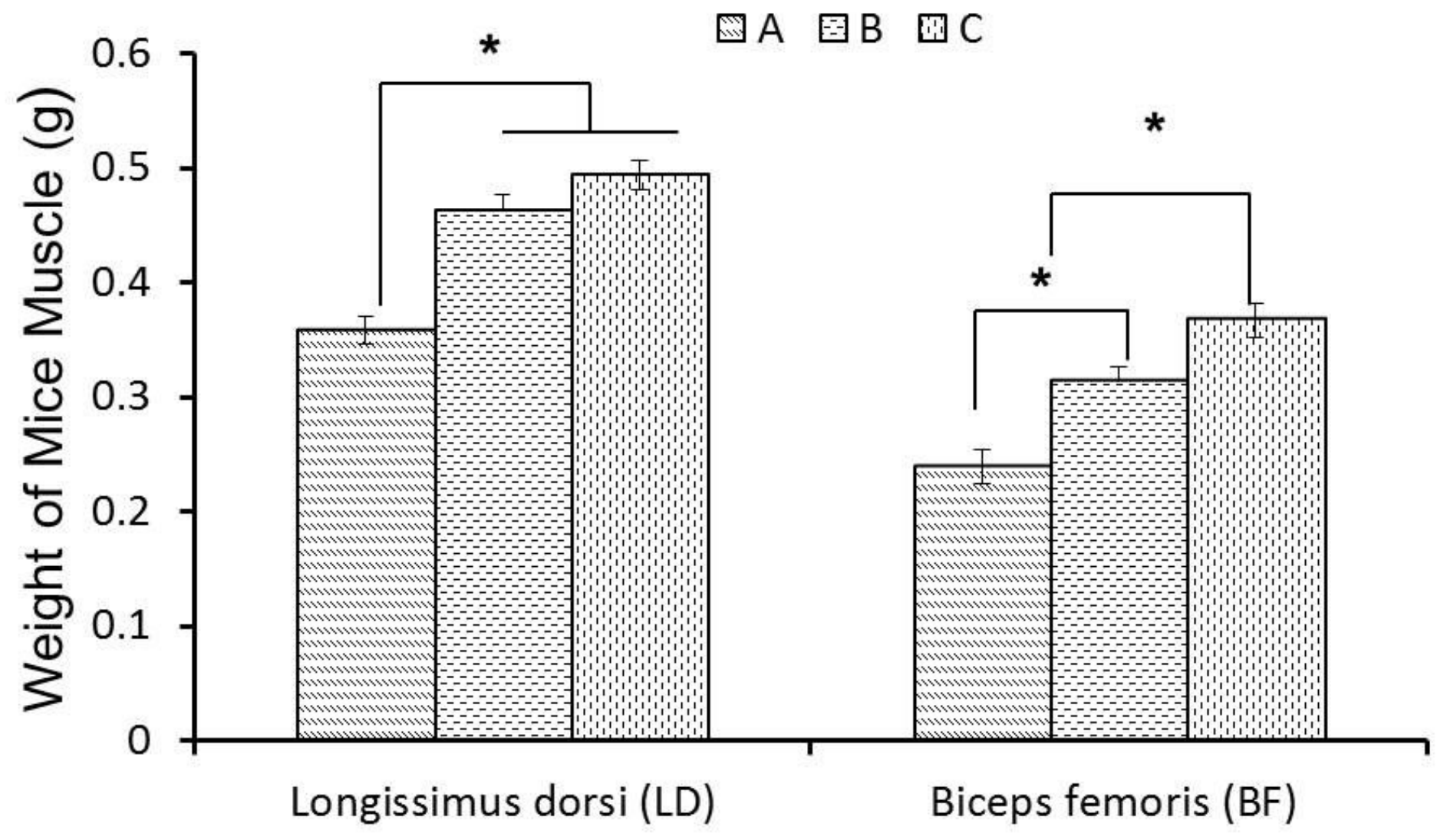

Figure 5

The weights of Longissimus dorsi (LD) and Biceps femoris (BF) of mice from different groups. LD and BF from each mouse $(n=10)$ were collected and weighted. Immunization significant increases the size of these two types of muscle. The date collected from group $A, B$, and $C$ were shown as $A, B$ and $C$ in the figure. Data are shown as Mean $\pm S D$. " * " was used to show the significant difference $(\mathrm{p} \leq 0.05)$. 


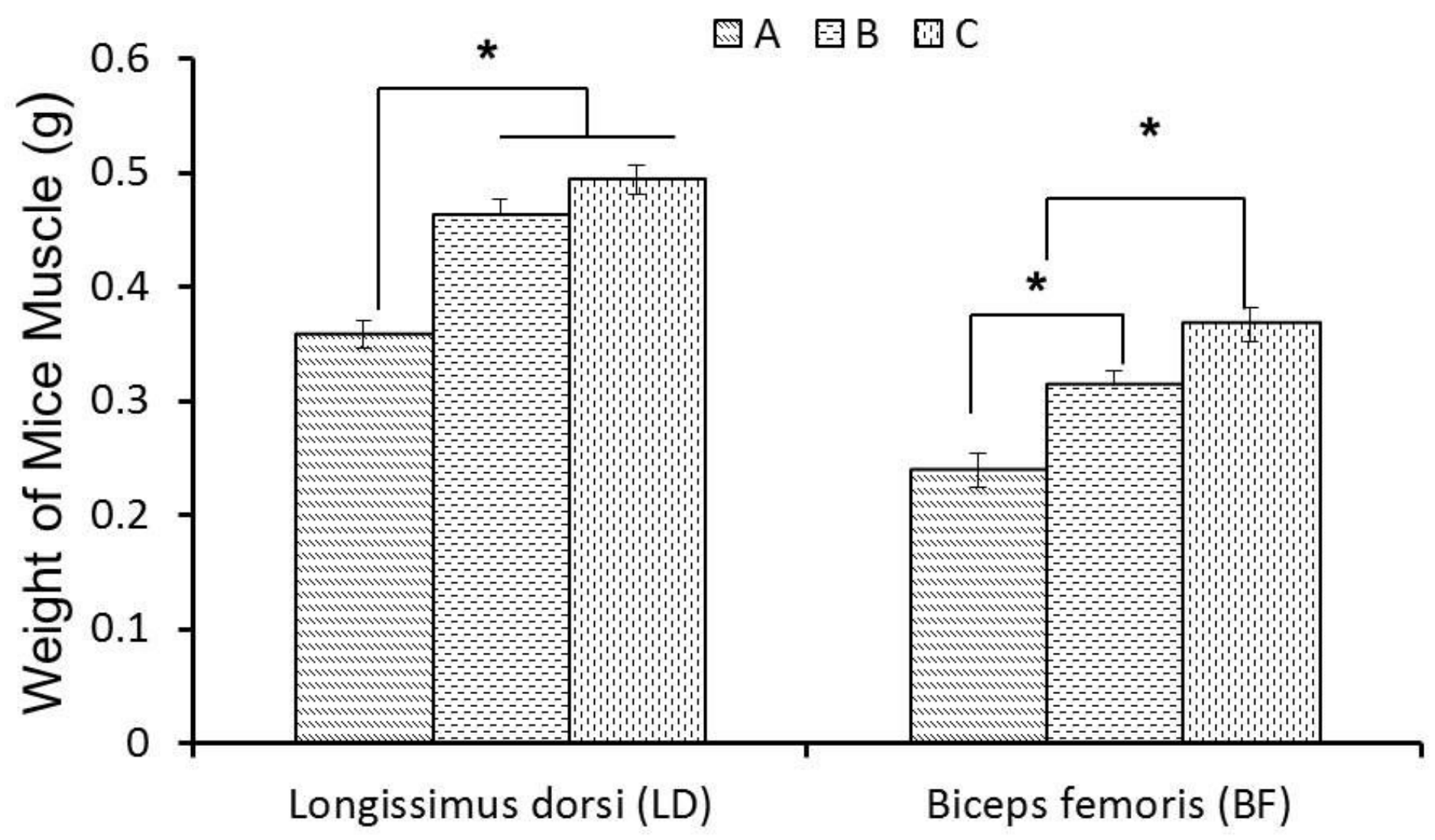

Figure 5

The weights of Longissimus dorsi (LD) and Biceps femoris (BF) of mice from different groups. LD and BF from each mouse $(n=10)$ were collected and weighted. Immunization significant increases the size of these two types of muscle. The date collected from group $A, B$, and C were shown as A, B and C in the figure. Data are shown as Mean \pm S D. " * " was used to show the significant difference $(p \leq 0.05)$.

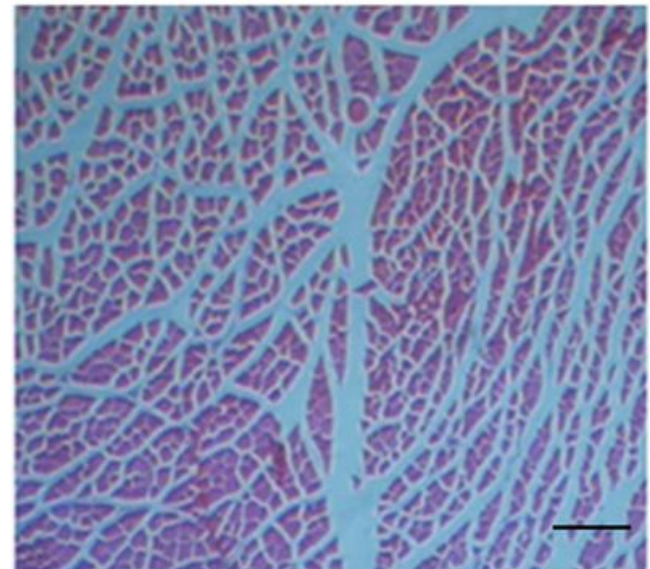

A

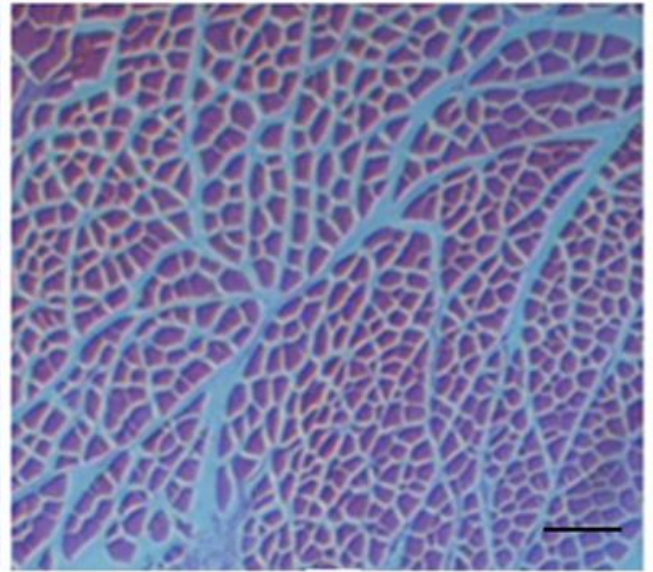

B

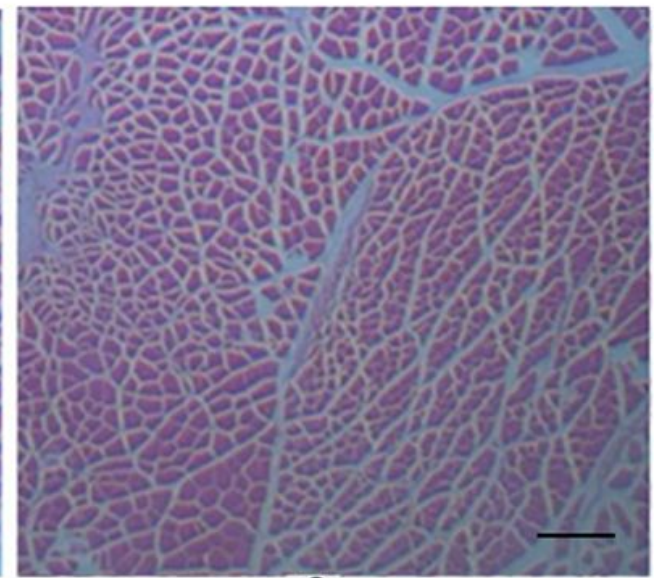

C

\section{Figure 6}

Histological investigation to examine muscle fibers of the mice. BF collected from each group were fixed and transected to make slices. The slice from group $A, B$, and $C$ were shown as $A, B$ and $C$ in the figure. The muscle fibers were observed under 


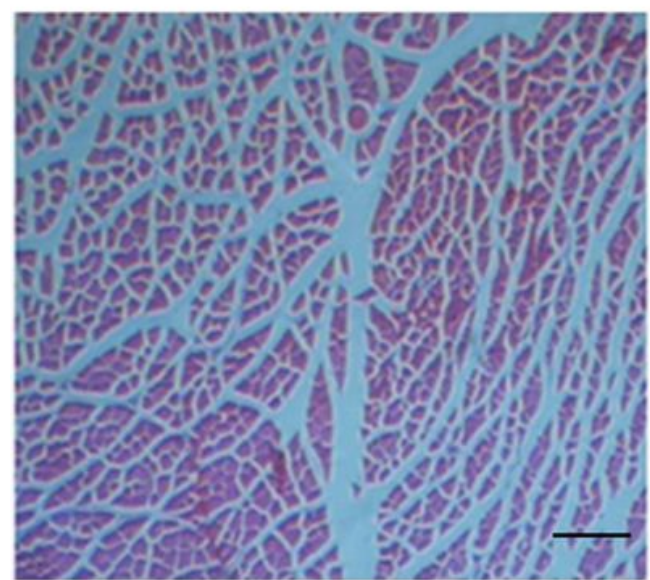

A

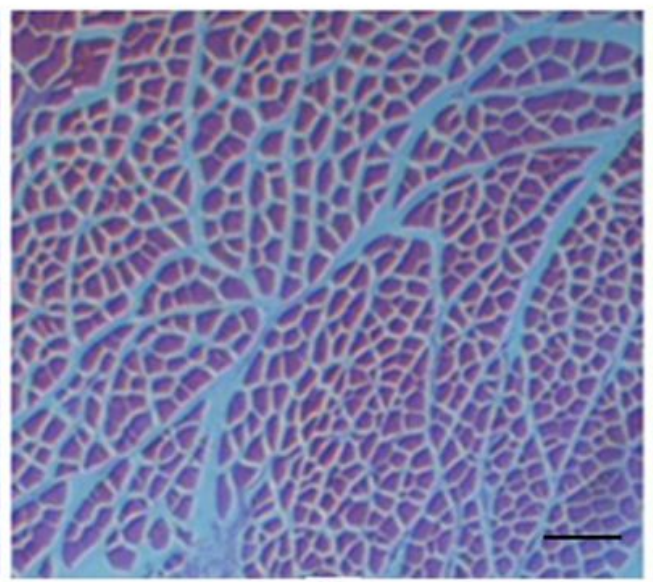

B

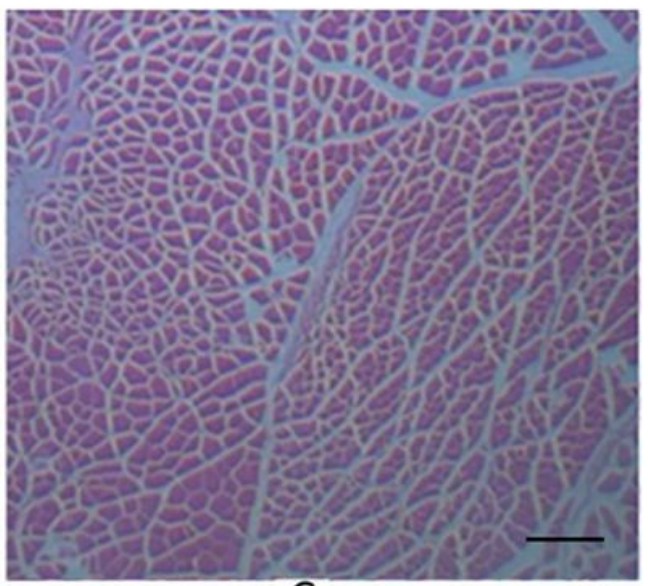

C

\section{Figure 6}

Histological investigation to examine muscle fibers of the mice. BF collected from each group were fixed and transected to make slices. The slice from group $A, B$, and $C$ were shown as $A, B$ and $C$ in the figure. The muscle fibers were observed under microscope. The length of the bar is $100 \mu \mathrm{m}$. The increased muscle fiber densities were observed in immunized groups.

\section{Supplementary Files}

This is a list of supplementary files associated with this preprint. Click to download.

- ARRIVEchecklist.docx

- ARRIVEchecklist.docx

- esupplementary.pdf

- esupplementary.pdf 